\title{
Dispositional Mindfulness and Past-Negative Time Perspective: The Differential Mediation Effects of Resilience and Inner Peace in Meditators and Non-Meditators
}

This article was published in the following Dove Press journal:

Psychology Research and Behavior Management

\author{
Jingjing $\mathrm{Ge} \mathbb{D}^{1,2}$ \\ Jingjing Yang $\mathbb{D D}^{3}$ \\ Jingjing Song' \\ Guangyu Jiang (D) ${ }^{1,2}$ \\ Yong Zheng (D' \\ 'Faculty of Psychology, Southwest \\ University, Chongqing, People's Republic \\ of China; ${ }^{2}$ Department of Humanities and \\ Management, Guizhou University of \\ Traditional Chinese Medicine, Guiyang, \\ Guizhou, People's Republic of China; \\ ${ }^{3}$ Department of Public Health and \\ Management, Chongqing Medical \\ University, Chongqing, People's Republic \\ of China
}

\begin{abstract}
Purpose: Past-negative time perspective (PNTP) can affect our everyday lives and is associated with negative emotions, unhealthy behaviors, rumination, anxiety, depression, and post-traumatic stress disorder (PTSD). Dispositional mindfulness may be able to reduce the negative effects of PNTP; however, few studies have investigated their relationship. Thus, the purpose of this study was to explore the effect dispositional mindfulness has on PNTP, as well as the mediating role of resilience and inner peace in this regard.

Methods: This study investigated the cross-sectional relationship between self-reported mindfulness, resilience, inner peace, and PNTP. In order to further explore the relationship between mindfulness and PNTP, this study specially selected and analyzed the samples of 185 meditators and 181 non-meditators.

Results: Correlation analysis revealed that mindfulness is significantly positively correlated with resilience and inner peace. Conversely, PNTP is significantly negatively correlated with mindfulness, resilience, and inner peace. Structural equation model analysis revealed that resilience and inner peace partially mediated the relationship between mindfulness and PNTP. Furthermore, a multi-group analysis showed that the mediating effects are different between meditators and non-meditators. For meditators, the effect of mindfulness on PNTP was fully mediated by resilience and inner peace. For non-meditators, the effect of mindfulness on PNTP was only partially mediated by resilience and inner peace.

Conclusion: Based on the significant differences between the mediational models of meditators and non-meditators, we believe that dispositional mindfulness can negatively predict PNTP, and practicing meditation consistently improves dispositional mindfulness, resilience and inner peace and effectively reduces PNTP. Our findings indicate that a combination of mindfulness and PNTP could be used to design new psychological interventions to reduce the symptoms of mental health concerns such as negative bias, rumination, depression, anxiety, and PTSD.
\end{abstract}

Keywords: psychological intervention, resilience, meditation, past-negative time perspective, unhealthy behaviors, inner peace

\section{Introduction}

Past-negative time perspective (PNTP) describes a generally pessimistic, negative, or aversive view and attitude toward the past. ${ }^{1}$ Due to the reconstructive nature of past experiences, these negative views and attitudes may be due to actual experiences of unpleasant or traumatic past events, negative reconstruction of benign past
Faculty of Psychology, Southwest University, Chongqing, People's Republic of China

Tel +86 I3667636|67

$\mathrm{Fax}+862368254139$

Email zhengy@swu.edu.cn 
events, or a mix of both. Several studies have shown that PNTP is positively associated with neuroticism, ${ }^{2}$ and anxiety, ${ }^{1}$ but is negatively associated with emotional stability, ${ }^{1}$ self-esteem, ${ }^{3}$ and subjective wellbeing. ${ }^{3,4}$

Although PNTP can affect routine cognitive and behavioral decisions, in general, the negative impact PNTP can have on one's life is controllable. However, some people with high PNTP are prone to engage in unhealthy behaviors that have a negative impact on their lives, such as excessive drinking or Internet addiction., ${ }^{5,6}$ Furthermore, some people who experienced negative or traumatic events may even "get stuck in the past," increasing the likelihood they will develop symptoms of post-traumatic stress disorder (PTSD), ${ }^{7,8}$ and depression. ${ }^{9,10}$

Several recent studies have identified a negative association between dispositional mindfulness and PNTP.,4,11 People with high dispositional mindfulness generally display less PNTP. ${ }^{12}$ Generally speaking, mindfulness is considered to be nonjudgmental acceptance of and nonreactive awareness to internal and external stimuli, including emotions, cognitions, and sensory input, as experienced in the present moment. ${ }^{13,14}$ Dispositional mindfulness is considered to be a psychological trait, and describes one's tendency to be mindful in everyday life. ${ }^{14-16}$ In recent decades, mindfulness meditation has received a great deal of application and research, these studies have shown that for clinical patients and healthy people alike, mindfulness training can significantly improve both physical and mental health, as well as promote overall psychological wellbeing. We posit that dispositional mindfulness may not only be negatively correlated with PNTP, but may also negatively predict PNTP. While PNTP reflects an orientation of attention toward the past with a pessimistic and negative attitude, ${ }^{1}$ mindfulness reflects an orientation of attention toward the present with a neutral and compassionate attitude. ${ }^{12,17}$ Mindfulness involves maintaining a conscious awareness of the present moment, which can adjust one's attention to time, improve one's attitude toward time, and make one's perspective of time more flexible. Thus, we propose hypothesis one: dispositional mindfulness can affect PNTP.

We posit that mindfulness can not only negatively affect PNTP directly, but also may negatively affect PNTP indirectly through resilience. Resilience is a psychological trait that helps individuals who have experienced adversity and trauma adjust and develop during trying circumstances, ${ }^{15}$ thus encouraging successful coping through "self-righting" ${ }^{18,19}$ It is a protective factor and developmental asset that can help people improve their subjective wellbeing. ${ }^{18}$ Studies have shown that dispositional mindfulness can be a significant predictor of resilience. ${ }^{15,20}$ Some previous studies have found that both dispositional mindfulness and resilience can effectively reduce the symptoms of mental health concerns such as negative bias, ${ }^{21,22}$ rumination, ${ }^{14,23}$ depression, ${ }^{13,24,25}$ anxiety, ${ }^{13,26}$ and PTSD. ${ }^{19,25}$ The IAA model of mindfulness suggests that mindfulness can enhance self-regulation and increase cognitive, emotional and behavioral flexibility, ${ }^{25,27}$ which may increase resilience and enhance adaptive coping to stressful events. ${ }^{20}$ Mindfulness and resilience can reduces these negative symptoms, thereby also reducing PNTP. Thus, we propose hypothesis two: resilience plays a mediating role in the relationship between dispositional mindfulness and PNTP.

In addition to resilience, we also believe that inner peace can play a mediating role in the relationship between dispositional mindfulness and PNTP. First of all, Xu et $\mathrm{al}^{28}$ consider inner peace, a relatively mild positive emotion, to represent calmness and harmony in the mind. Inner peace was found to significantly decrease symptoms of depression and anxiety; therefore, psychology researchers usually regard inner peace as an important indicator of mental health. ${ }^{29}$ Lee et $\mathrm{al}^{29}$ further found that compared to Americans, Chinese individuals scored higher on scales used to measure inner peace. This indicates that in Chinese culture, individuals are more focused on inner peace as a core principle in the pursuit of emotional wellbeing. Xu et al ${ }^{28}$ also identified "peace of mind," as understood in Chinese culture, to be a main component of the Eastern cultural concept of subjective wellbeing. Secondly, in ancient Buddhism, achieving inner peace is the core goal of meditation. ${ }^{30}$ Empirical studies have shown that inner peace remains an important goal in contemporary meditation as well. ${ }^{31,32}$ Recent studies have demonstrated that dispositional mindfulness is also a significant predictor of experiencing inner peace. ${ }^{28}$ Meditation's fourfold model of well-being suggests that mindfulness can improve an individual's ability to regulate emotions. ${ }^{33}$ Mindfulness encourages facing one's own experiences with acceptance and a non-judgmental attitude in the moment. When a person non-judgmentally accepts him/herself instead of blaming or criticizing his/her feelings, emotions, and thoughts, it becomes easier to maintain and enhance emotional balance and inner peace. ${ }^{28,31}$ According to the broaden-and-build theory of positive emotions, ${ }^{34}$ positive emotions can broaden the scope of cognition, enhance cognitive flexibility, and promote effective problem solving. It is speculated that inner peace could help people reduce negative cognition and increase positive emotions, which 
could consequently encourage individuals to develop a more flexible view of life and time, thereby reducing PNTP. Thus, we propose hypotheses three: inner peace plays a mediating role in the relationship between dispositional mindfulness and PNTP.

In addition, recent research has found that dispositional mindfulness not only directly affects inner peace but also indirectly affects inner peace through self-acceptance. ${ }^{28}$ Recent studies have provided evidence that individuals with high resilience also experience more equanimity and peacefulness when facing stressful events. ${ }^{20}$ We believe that mindfulness can also cultivate and improve inner peace through resilience. Mindfulness can improve resilience, and resilience may improve inner peace by improving emotional regulation and cognitive flexibility. ${ }^{20,25}$ Thus, we propose hypotheses four: resilience and inner peace plays a serial mediating role in the relationship between mindfulness and PNTP.

At last, Pepping et $\mathrm{al}^{35}$ found that experienced meditators have significantly higher levels of dispositional mindfulness than inexperienced meditators. Wittmann et $\mathrm{al}^{36}$ found that the PNTP scores in a long-term meditator group was significantly lower than in the control group (non-meditators). In addition, Liu et $\mathrm{al}^{31}$ found that eight weeks of mindfulness training led to a significant increase in scores for mindfulness and inner peace in the meditation group, compared with the control group (non-mindfulness intervention). After a brief mindfulness training, the mindfulness and resilience of the participants were significantly improved. ${ }^{37}$ Since these variables may be different between meditators and non-meditators in the present study. Thus, we propose hypothesis five: the serial mediating role of resilience and inner peace affecting dispositional mindfulness and PNTP may be different in meditators and nonmeditators.

\section{Materials and Methods}

\section{Participants}

In total, questionnaires were distributed to 416 participants in the meditator group and the working population through an Internet platform; however, due to incomplete information about meditation practice, data of 50 participants were excluded from analysis. Thus, a total of 366 individuals (152 female; 214 male) completed the questionnaire and were included in the final study sample. The final analysis sample had an overall age range of 18 to 55 years with a mean age of 33.71 years $(\mathrm{SD}=7.81)$. The education level of the sample was generally high, with $81.15 \%$ of the participants having completed university studies. Of the participants, 185 (75 female; 110 male) regularly engaged in meditation and had an overall age range of 18 to 55 years, with a mean age of 34.41 years $(\mathrm{SD}=8.07)$. The other participants were 181 individuals (77 female; 104 male) who had never regularly meditated (although they may have tried it once or on a few occasions) and had an overall age range of 19 to 54 years, with a mean age of 32.99 years $(\mathrm{SD}=7.49)$.

\section{Procedure}

The present study was approved by the ethics committee of the Faculty of Psychology at Southwest University, China. All participants gave online informed consent before completing the questionnaire. Respondents were told that the questionnaire was entirely anonymous, and that they would receive feedback regarding their results.

\section{Measures}

\section{Meditation Experience Information}

Meditation experience was assessed with a brief questionnaire designed by Baer et al. ${ }^{38}$ Among the regular meditators, mean lifetime duration of meditation practice was 616.51 hours ( $\mathrm{SD}=845.294)$, and ranged among participants from 20 hours to 3000 hours. More than half $(55.68 \%)$ reported meditating more than three times per week. Meditation practice was defined broadly, and included Vipassana meditation, loving kindness meditation, walking meditation, and yoga.

\section{Measurement of Dispositional Mindfulness}

Dispositional mindfulness was measured using the Chinese version of the Mindful Attention Awareness Scale (MAAS). ${ }^{39}$ The MAAS consists of one dimension and 15 items rated on a 6-point scale ranging from 1 ("almost always") to 6 ("almost never"). Previous research found that the MAAS has good reliability and validity. ${ }^{39}$ In the present study, the reliability coefficient of the whole scale was 0.88 .

\section{Measurement of Resilience}

Resilience was assessed using the Chinese version of the Connor-Davidson Resilience Scale (CD-RISC), as translated and revised by $\mathrm{Yu}$ and Zhang. ${ }^{40}$ The CD-RISC contains 25 items and divides resilience into three dimensions: fortitude, self-improvement, and optimism. The CD-RISC rates each item on a 5-point scale ranging from 0 ("not true at all") to 5 ("true nearly all of the time"), with higher scores reflecting greater resilience. The Chinese version of 
the CD-RISC has been shown to have good reliability and validity. ${ }^{40}$ In the present study, the reliability coefficient of the whole scale was 0.90 , and the reliability coefficients for the subscales were 0.84 for fortitude, 0.70 for selfimprovement, and 0.63 for optimism.

\section{Measurement of Inner Peace}

The Peace of Mind Scale (PoM) was used to measure peace of mind. ${ }^{29}$ It comprises a total of 7 items rated on a 5-point scale ranging from 1 ("not at all") to 5 ("all of the time"). Previous research found that the PoM has good reliability and validity. ${ }^{29}$ Cronbach's alpha in the present study was 0.89 .

\section{Measurement of Past-Negative Time Perspective}

PNTP was measured using the past-negative dimension of the Zimbardo Time Perspective Inventory (ZTPI). ${ }^{1}$ The ZTPI has shown good cross-cultural reliability and validity. ${ }^{41}$ The past-negative dimension of the ZTPI comprises 10 items rated on a 5-point Likert scale. In the present study, the reliability coefficient of the PNTP scale was 0.83 .

\section{Statistical Analyses}

All analyses in this study were made using the SPSS 23.0 and AMOS 23.0 software programs. First, we examined the descriptive statistics and correlations of the study variables using SPSS 23.0. Second, AMOS 23.0 was used to establish structural equation models and examine the mediating effects and differences in the totality of the meditator and non-meditator groups.

\section{Results}

\section{Common Method Bias Test}

After the data collection was completed, a Harman single factor test was used to test common method bias. The results showed that the first factor only accounted for $21.99 \%$ of variance, which was below the critical standard of $40 \%$, indicating that common method bias was not significant.

\section{Descriptive Statistics and Correlation Analysis of Each Variable}

The results, as shown in Table 1, demonstrate that mindfulness is significantly positively correlated with resilience and inner peace. Conversely, PNTP is negatively correlated with mindfulness, resilience, and inner peace.
Table I Correlations Between Mindfulness, Resilience, Inner Peace, and PNTP

\begin{tabular}{|l|l|l|l|l|l|l|}
\hline & Mean & SD & $\mathbf{I}$ & $\mathbf{2}$ & $\mathbf{3}$ & $\mathbf{4}$ \\
\hline I. Mindfulness & 3.82 & 0.75 & - & & & \\
2. Resilience & 2.45 & $0.5 \mathrm{I}$ & $0.37^{* *}$ & - & & \\
3. Inner peace & 3.33 & 0.69 & $0.38^{* *}$ & $0.56^{* *}$ & - & \\
4. PNTP & 2.84 & 0.70 & $-0.4 \mathrm{I}^{* *}$ & $-0.4 \mathrm{I}^{* *}$ & $-0.49 * *$ & - \\
\hline
\end{tabular}

Notes: $\mathrm{n}=366,{ }^{* *} p<0.01$.

Abbreviation: PNTP, past-negative time perspective.

\section{$t$-Test for Differences Between Meditator and Non-Meditator Groups}

After independent sample $t$-test and chi-square test, the results are shown in Table 2, no significant differences in gender, age, educational background or economic income between The two groups.

As shown in Table 3, results of the $t$-test indicated that meditators and non-meditators show significant differences in mindfulness, inner peace, and PNTP.

\section{Testing the Multiple Mediation Model of Dispositional Mindfulness Influencing Past-Negative Time Perspective}

$\mathrm{Wu}$ and $\mathrm{Wen}^{42}$ suggest that a single dimensional scale should be parceled. In the present study, these parcels

Table 2 t-Test and Chi-Square Test of Major Demographic Variables Between the Meditation Group and the NonMeditation Group

\begin{tabular}{|c|c|c|c|c|}
\hline & $\begin{array}{l}\text { Meditator } \\
\text { Group } \\
(n=185)\end{array}$ & $\begin{array}{l}\text { Non-Meditator } \\
\text { Group }(n=181)\end{array}$ & t/F-value & $P$ \\
\hline $\begin{array}{l}\text { Age (years) } \\
(\text { Mean } \pm S D)\end{array}$ & $34.41 \pm 8.069$ & $32.99 \pm 7.491$ & 1.739 & 0.083 \\
\hline $\begin{array}{l}\text { Gender } \\
\text { Male } \\
\text { Female }\end{array}$ & $\begin{array}{l}110 \\
75\end{array}$ & $\begin{array}{l}104 \\
77\end{array}$ & 0.151 & 0.698 \\
\hline $\begin{array}{l}\text { Educational } \\
\text { situation } \\
\text { Junior college } \\
\text { and below } \\
\text { University } \\
\text { Postgraduate }\end{array}$ & $\begin{array}{l}38 \\
78 \\
69\end{array}$ & $\begin{array}{l}31 \\
97 \\
53\end{array}$ & 4.828 & 0.089 \\
\hline $\begin{array}{l}\text { Monthly income } \\
\text { (RMB) } \\
<3000 \\
3000 \sim 6000 \\
>6000\end{array}$ & $\begin{array}{l}21 \\
106 \\
58\end{array}$ & $\begin{array}{l}16 \\
123 \\
42\end{array}$ & 4.455 & 0.108 \\
\hline
\end{tabular}


Table 3 Group Differences Between Meditators and NonMeditators in Mindfulness, Resilience, Inner Peace, and PNTP

\begin{tabular}{|c|c|c|c|c|}
\hline & $\begin{array}{l}\text { Meditator } \\
\text { Group } \\
\text { Mean } \pm \text { SD } \\
(n=185)\end{array}$ & $\begin{array}{l}\text { Non-Meditator } \\
\text { Group } \\
\text { Mean } \pm \text { SD } \\
(n=18 I)\end{array}$ & $t$ & $P$ \\
\hline Mindfulness & $4 . I I \pm 0.73$ & $3.52 \pm 0.66$ & $8.12^{* * *}$ & 0.000 \\
\hline Resilience & $2.49 \pm 0.5 \mathrm{I}$ & $2.40 \pm 0.50$ & 1.57 & 0.116 \\
\hline Inner peace & $3.42 \pm 0.69$ & $3.24 \pm 0.68$ & $2.50 *$ & 0.013 \\
\hline PNTP & $2.73 \pm 0.75$ & $2.95 \pm 0.63$ & $-2.99 * *$ & 0.003 \\
\hline
\end{tabular}

Notes: $\mathrm{n}=366, * p<0.05, * * p<0.01, * * * p<0.001$.

Abbreviation: PNTP, past-negative time perspective.

were formed to control for inflated measurement errors caused by multiple items for the latent factor. Three item parcels each were created for mindfulness and PNTP and two item parcels were created for inner peace. A random algorithm was used to combine the items into parcels. In addition, the variables of gender and age were controlled in the subsequent structural equation model analysis. Next, we created a multiple mediation model to examine whether resilience and inner peace mediate the impact of dispositional mindfulness on PNTP, and, if so, how this is done. Finally, AMOS 23.0 was used to perform bootstrapping procedures to test the significance of the mediation effects of resilience and inner peace. We generated 2000 bootstrapping samples from the original dataset $(\mathrm{N}=366)$ through random sampling. Empirical 95\% confidence intervals did not contain zero, indicating that the indirect effect was statistically significant.

As shown in Figure 1 and Table 4, the bootstrap method revealed that both resilience and inner peace mediate the association between dispositional mindfulness and PNTP. The significant mediating roles were: (a) that of resilience for PNTP, accounting for $12.95 \%$ of the total effect $(95 \% \mathrm{CI}$ $[-0.096,-0.001])$; (b) that of inner peace for PNTP,

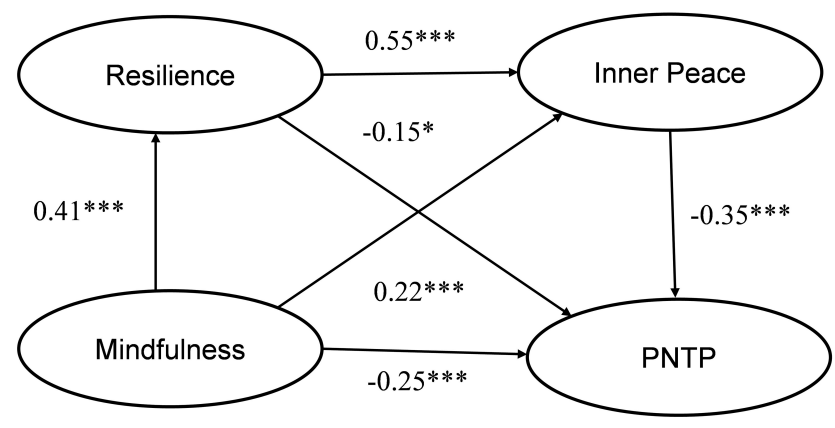

Figure I The multiple mediator model of mindfulness on PNTP. Notes: ${ }^{*} p<0.05,{ }^{* * *} p<0.001$.

Abbreviation: PNTP, past-negative time perspective.
Table 4 Bootstrapping Indirect Effects and 95\% Confidence Intervals $(\mathrm{Cl})$ for the Mediation Model

\begin{tabular}{|l|l|l|}
\hline Model Pathways & Effect Size & Bias-Corrected Cl \\
\cline { 2 - 2 } & Standardized & (95\%) \\
& $\boldsymbol{\beta}$ & \\
\hline Mindfulness $\rightarrow$ resilience $\rightarrow$ PNTP & $-0.06^{*}$ & {$[-0.096,-0.001]$} \\
Mindfulness $\rightarrow$ inner peace $\rightarrow$ PNTP & $-0.08^{* *}$ & {$[-0.102,-0.023]$} \\
Mindfulness $\rightarrow$ resilience $\rightarrow$ inner & $-0.08^{* * *}$ & {$[-0.102,-0.031]$} \\
peace $\rightarrow$ PNTP & & \\
\hline
\end{tabular}

Notes: $\mathrm{n}=366,{ }^{*} p<0.05, * * p<0.01, * * * p<0.001$

Abbreviation: PNTP, past-negative time perspective.

accounting for $16.35 \%$ of the total effect ( $95 \%$ CI $[-0.102$, $-0.023]$ ); and (c) the serial mediating role of resilience via inner peace for PNTP, which accounted for $16.99 \%$ of the total effect $(95 \%$ CI $[-0.102,-0.031])$. Therefore, our findings also support hypotheses 1,2, 3, and 4 .

\section{Testing Group Differences Between Meditators and Non-Meditators in the Multiple Mediation Model}

First, the mediation effect model was tested on the meditator and non-meditator groups, respectively. The fit indices of the obtained models are shown in Table 5 . Both the meditator model $\left(\mathrm{M}_{\mathrm{m}}\right)$ and the non-meditator model $\left(\mathrm{M}_{\mathrm{n}}\right)$ were acceptable and can be compared across groups.

Next, using the multi-group comparison method of structural equation modeling, the unconstrained model $\left(\mathrm{M}_{1}\right)$ and the structural covariances model $\left(\mathrm{M}_{2}\right)$ were set. The fitting results of the two models are shown in Table 5 wherein a significant difference between $\mathrm{M}_{1}$ and $\mathrm{M}_{2}\left(\Delta \chi^{2}\right.$ $=52.18, \Delta d f=19, p<0.001)$ is indicated. These findings support hypothesis 5 .

As shown in Figures 2 and 3 and Table 6, the bootstrap method was used to test the mediation effects of the meditator and non-meditator models. In the meditator group, the serial mediating role of resilience and inner peace between mindfulness and PNTP accounted for $23.44 \%$ of the total effect ( $95 \%$ CI $[-0.188,-0.046]$ ), and the serial mediating effects in the non-meditator group accounted for $10.98 \%$ of the total effect $(95 \%$ CI $[-0.109,-0.007])$.

In the meditator group, resilience and inner peace had significant intermediation between mindfulness and PNTP, respectively. Resilience for PNTP accounted for 36.31\% of the total effect $(95 \%$ CI $[-0.281,-0.065])$, and inner 
Table 5 Comparison of the Difference Between Meditator and Non-Meditator Groups in the Serial Mediation Models

\begin{tabular}{|l|l|l|l|l|l|l|l|}
\hline Model & $\chi^{2}$ & df & RMSEA & GFI & NFI & TLI \\
\hline$M_{m}$ & 98.936 & 58 & 0.062 & 0.927 & 0.927 & 0.957 & 0.968 \\
$M_{n}$ & 111.915 & 58 & 0.072 & 0.918 & 0.884 & 0.918 & 0.939 \\
$M_{1}$ & 210.852 & 116 & 0.047 & 0.923 & 0.909 & 0.941 & 0.956 \\
$M_{2}$ & 263.033 & 135 & 0.051 & 0.905 & 0.886 & 0.931 & 0.941 \\
\hline
\end{tabular}

Abbreviations: $M_{m}$, the meditator model; $M_{n}$, the non-meditator model; $M_{1}$, the unconstrained model; $M_{2}$, the structural covariances model.

peace for PNTP accounted for $40.25 \%$ of the total effect (95\% CI $[-0.322,-0.081])$. The non-meditator group did not display these two effects.

\section{Discussion}

The Multiple Mediation Model of Dispositional Mindfulness Influencing Past-Negative Time Perspective in the Whole Group

Our findings show that dispositional mindfulness negatively affects PNTP. On the one hand, dispositional

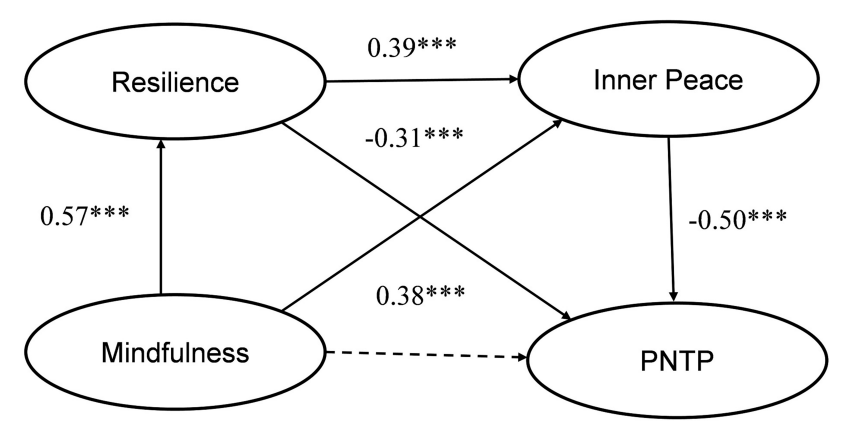

Figure 2 The multiple mediator model of mindfulness on PNTP (meditator group). Notes: The dashed lines in the figure indicate that the path coefficient was not significant; $* * * p<0.00$ I.

Abbreviation: PNTP, past-negative time perspective.

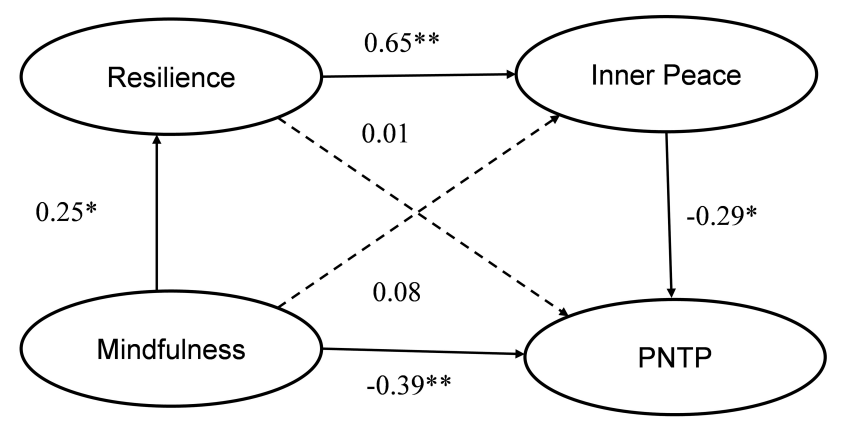

Figure 3 The multiple mediator model of mindfulness on PNTP (non-meditator group).

Notes: The dashed lines in the figure indicate that the path coefficient was not significant; $*_{p}<0.05,{ }^{*} p<0.01$.

Abbreviation: PNTP, past-negative time perspective. mindfulness can negatively predict PNTP, and previous studies have found a negative correlation between dispositional mindfulness and PNTP. ${ }^{4,11}$ However, our research is a step forward from these studies as they have not further explored the relationship between dispositional mindfulness and PNTP. Mindfulness may be able to directly affect PNTP because mindfulness is a way of balancing awareness, encouraging one to observe thoughts and feelings without avoiding or attempting to change them or applying exaggeration or bias. ${ }^{33}$ This style of awareness can help reduce self-criticism, avoid overthinking negative events, and protect individuals from the harmful effects of depression, anxiety, and other negative emotional states. ${ }^{13,14,39}$ Furthermore, as a result of the influences of culture, education, religion, social class, and family, humans can rely too much on a particular time perspective, and this behavior becomes automatic. ${ }^{1}$ Mindfulness has a de-automating effect that can reduce tendencies towards cognitive behavioral habituation, ${ }^{43}$ helping to reduce automatic or habitual overuse of a particular time perspective.

On the other hand, dispositional mindfulness can also indirectly affect PNTP through resilience or inner peace. This is consistent with the IAA model of mindfulness which indicates that mindfulness involves a nonjudgmental awareness and acceptance of the present experience. ${ }^{21,27}$ This enhances individuals' cognitive functioning and emotional regulation, thereby improving resilience and enhancing inner peace, and may, therefore provide a more balanced and flexible time perspective.

\section{The Differences in Multiple Mediation Models of Dispositional Mindfulness Influencing PNTP in the Meditator and Non-Meditator Groups}

The present study found that for the meditator group, the influence of dispositional mindfulness on PNTP was completely mediated by resilience and inner peace. For the nonmeditator group, only a small part of the influence of dispositiona mindfulness on PNTP was mediated by 
Table 6 Bootstrapping Indirect Effects and 95\% Confidence Intervals (Cl) for the Mediation Model in Meditators and Non-Meditators

\begin{tabular}{|c|c|c|c|c|}
\hline \multirow[t]{2}{*}{ Model Pathways } & \multicolumn{2}{|c|}{ Meditator Group $(n=185)$} & \multicolumn{2}{|c|}{ Non-Meditator Group $(n=\mid 81)$} \\
\hline & $\begin{array}{l}\text { Effect Size } \\
(\text { Standardized } \beta)\end{array}$ & $\begin{array}{l}\text { Bias-Corrected Cl } \\
(95 \%)\end{array}$ & $\begin{array}{l}\text { Effect Size } \\
(\text { Standardized } \beta)\end{array}$ & $\begin{array}{l}\text { Bias-Corrected Cl } \\
(95 \%)\end{array}$ \\
\hline Mindfulness $\rightarrow$ resilience $\rightarrow$ PNTP & $-0.175^{* *}$ & {$[-0.281,-0.065]$} & 0.003 & {$[-0.052,0.054]$} \\
\hline Mindfulness $\rightarrow$ inner peace $\rightarrow$ PNTP & $-0.194^{* * *}$ & {$[-0.322,-0.081]$} & -0.025 & {$[-0.073,0.008]$} \\
\hline $\begin{array}{l}\text { Mindfulness } \rightarrow \text { resilience } \rightarrow \text { inner } \\
\text { peace } \rightarrow \text { PNTP }\end{array}$ & $-0.113^{* * *}$ & {$[-0.188,-0.046]$} & $-0.048^{*}$ & {$[-0.109,-0.007]$} \\
\hline
\end{tabular}

Notes: $*_{p}<0.05, * *_{p}<0.01, * * * p<0.001$.

Abbreviation: PNTP, past-negative time perspective.

resilience and inner peace. This may be because in the meditator group, due to the regular and continuous practice of meditation, resilience and inner peace have gradually become internalized as relatively stable psychological qualities. This is consistent with previous studies showing that mindfulness meditation can cultivate qualities such as loving-kindness, compassion, inner peace, and resilience. ${ }^{20,33}$ Additionally, Buddhism has a system of meditative practices for cultivating similar qualities, further illustrating the importance of meditation practice. Mindfulness meditation has always emphasized that only by the consistent practicing of mindfulness skills can these qualities be cultivated, maintained, and improved.

Additionally, the present study found that for the meditator group, the effect of inner peace was greater than that of resilience, suggesting that inner peace may be an important predictor of and a good representation of Chinese emotional wellbeing. This finding is consistent with previous research. ${ }^{28,31}$ According to Lee et al, ${ }^{29}$ traditional Chinese culture is greatly influenced by the three main religions or philosophies of Taoism, Confucianism, and Buddhism, which all value and emphasize inner peace. Additionally, mindfulness can promote inner peace, which is both consistent with previous research and with Buddhist thought. ${ }^{28,31}$ The ultimate goal of meditation is inner peace, and in Buddhism, inner peace is considered to be the most important thing in life..$^{30,31}$

Finally, the present study found that for nonmeditators, the direct effect of mindfulness on PNTP accounted for $89.04 \%$ of the total effect. Arguably, the direct effect of mindfulness on PNTP is relatively large, while the indirect effect of resilience and inner peace only provide a relatively small part of the total effect. This shows that mindfulness can promote psychological functioning and mental health, allowing individuals to better understand, manage, and solve problems in daily life, thereby reducing PNTP. However, the direct effect of mindfulness on PNTP also suggests that mindfulness may reduce PNTP in other ways as well. In addition to resilience and inner peace, there may be other mediating variables, which future research should work to identify.

\section{Conclusion and Limitations}

This study has a few limitations. Firstly, the results relied completely upon self-report data, which may have potential problems, such as recall bias or response bias. Also, mindfulness is a complex and abstract construct that involves an advanced level of meta-cognition, ${ }^{44}$ so self-report methods may not be a reliable source of information. ${ }^{45}$ Future research can add direct and objective assessment measures (e.g., the Triangle task) or qualitative analysis to supplement the assessment of mindfulness. ${ }^{45}$ Secondly, since this study adopted a cross-sectional design, it was not appropriate to make causal inferences. In addition, we took mindfulness as the independent variable and PNTP as the mediating variable, and took inner peace or resilience as the dependent variables to make structural equation models. Although the models are weak, they remain significant, thus, the interpretation proposed here should be treated with proper caution. Future studies should design experiments or longitudinal studies that could complement and validate our findings.

Additionally, a multi-group analysis showed that, for meditators, the effect of dispositional mindfulness on PNTP was fully mediated by resilience and inner peace; however, for non-meditators, the effect of mindfulness on PNTP was partially mediated by resilience and inner peace. Thus, the mediational model was significantly different between meditators and non-meditators. On the basis of these findings, we conclude that dispositional mindfulness, resilience, and inner peace all negatively predict PNTP. Finally, some studies have found that the psychological 
problems, such as emotional regulation, addiction, procrastination, anxiety, depression, can be relieved or reduced by regulating the individual's PNTP or by jointly regulating other dimensions of time perspectives. ${ }^{46,47}$ In the future, a combination of mindfulness and time perspective could be used to design new psychological interventions to regulate moods, manage addiction, reduce unhealthy behaviors, relieve stress and PTSD, and promote positive individual mental health outcomes.

\section{Acknowledgments}

The authors would like to thank the research assistants who undertook data collection and the participants for their selfless support of the study.

\section{Author Contributions}

All authors contributed to data analysis, drafting and revising the article, gave final approval of the version to be published, and agree to be accountable for all aspects of the work.

\section{Funding}

This research was supported by the MOE Project of Key Research Institute of Humanities and Social Sciences at Universities (15JJDZONGHE022), China.

\section{Disclosure}

The authors report no conflicts of interest in this work.

\section{References}

1. Zimbardo PG, Boyd JN. Putting time in perspective: a valid, reliable individual-differences metric. J Pers Soc Psychol. 1999;77 (6):1271-1288. doi:10.1037/0022-3514.77.6.1271

2. Sobol-Kwapinska M. Calm down-It's only neuroticism. Time perspectives as moderators and mediators of the relationship between neuroticism and well-being. Pers Indiv Differ. 2016;94:64-71. doi:10.1016/j.paid.2016.01.004

3. Seema R, Sircova A. Mindfulness-a time perspective? Estonian study. Baltic J Psychol. 2013;14:4-21.

4. Ge J, Wu J, Li K, Zheng Y. Self-compassion and subjective well-being mediate the impact of mindfulness on balanced time perspective in Chinese college students. Front Psychol. 2019;10:367. doi:10.3389/ fpsyg.2019.00367

5. Chavarria J, Allan NP, Moltisanti A, Taylor J. The effects of present hedonistic time perspective and past negative time perspective on substance use consequences. Drug Alcohol Depend. 2015;152:39-46. doi:10.1016/j.drugalcdep.2015.04.027

6. Przepiorka A, Blachnio A. Time perspective in internet and Facebook addiction. Comput Human Behav. 2016;60:13-18. doi:10.1016/j. chb.2016.02.045

7. Kazak AE, Boeving CA, Alderfer MA, Hwang WT, Reilly A. Posttraumatic stress symptoms during treatment in parents of children with cancer. J Clin Oncol. 2005;23(30):7405-7410. doi:10.1200/ JCO.2005.09.110
8. Stolarski M, Cyniak-Cieciura M. Balanced and less traumatized: balanced time perspective mediates the relationship between temperament and severity of PTSD syndrome in motor vehicle accident survivor sample. Pers Indiv Differ. 2016;101:456-461. doi:10.1016/j. paid.2016.06.055

9. Bitsko MJ, Stern M, Dillon R, Russell EC, Laver J. Happiness and time perspective as potential mediators of quality of life and depression in adolescent cancer. Pediatr Blood Cancer. 2008;50 (3):613-619. doi:10.1002/pbc.21337

10. Habermas T, Ott L, Schubert M, Schneider B, Pate A. Stuck in the past: negative bias, explanatory style, temporal order, and evaluative perspectives in life narratives of clinically depressed individuals. Depress Anxiety. 2008;25(11):121-132. doi:10.1002/da.20389

11. Drake L, Duncan E, Sutherland F, Abernethy C, Henry C. Time perspective and correlates of wellbeing. Time Soc. 2008;17 (1):47-61. doi:10.1177/0961463X07086304

12. Wittmann M, Peter J, Gutina O, Otten S, Kohls N, Meissner K. Individual differences in self-attributed mindfulness levels are related to the experience of time and cognitive self-control. Pers Indiv Differ. 2014;64:41-45. doi:10.1016/j.paid.2014.02.011

13. Brown KW, Ryan RM. The benefits of being present: mindfulness and its role in psychological well-being. J Pers Soc Psychol. 2003;84 (4):822-848. doi:10.1037/0022-3514.84.4.822

14. Josefsson T, Ivarsson A, Lindwall M, et al. Mindfulness mechanisms in sports: mediating effects of rumination and emotion regulation on sport-specific coping. Mindfulness. 2017;8(5):1354-1363. doi:10.10 07/s12671-017-0711-4

15. Bajaj B, Pande N. Mediating role of resilience in the impact of mindfulness on life satisfaction and affect as indices of subjective well-being. Pers Indiv Differ. 2016;93:63-67. doi:10.1016/j.paid. 2015.09.005

16. Short MM, Mazmanian D, Oinonen K, Mushquash CJ. Executive function and self-regulation mediate dispositional mindfulness and well-being. Pers Indiv Differ. 2016;93:97-103. doi:10.1016/j. paid.2015.08.007

17. Stolarski M, Vowinckel J, Jankowski KS, Zajenkowski M. Mind the balance, be contented: balanced time perspective mediates the relationship between mindfulness and life satisfaction. Pers Indiv Differ. 2016;93:27-31. doi:10.1016/j.paid.2015.09.039

18. Richardson GE. The metatheory of resilience and resiliency. $J$ Clin Psychol. 2002;58(3):307-321. doi:10.1002/jclp.10020

19. Zheng Y, Fan F, Yu C, Luo T.Relationship between gratitude and symptoms of post-traumatic stress disorder among adolescents: mediation of social support and resilience. Psychol Dev Educ. 2011;27 (05):522-528.

20. Epstein RM, Krasner MS. Physician resilience: what it means, why it matters, and how to promote it. Acad Med. 2013;88(3):301-303. doi:10.1097/ACM.0b013e318280cff0

21. Garland EL, Farb NA, Goldin P, Fredrickson BL. Mindfulness broadens awareness and builds eudaimonic meaning: a process model of mindful positive emotion regulation. Psychol Inq. 2015;26(4):293-314. doi:10. 1080/1047840X.2015.1064294

22. Kiken LG, Shook NJ. Looking up: mindfulness increases positive judgments and reduces negativity bias. Soc Psychol Pers Sci. 2010;2 (4):425-431. doi:10.1177/1948550610396585

23. Wolkin JR. Cultivating multiple aspects of attention through mindfulness meditation accounts for psychological well-being through decreased rumination. Psychol Res Behav Ma. 2015;8:171-180. doi:10.2147/PRBM.S31458

24. Edenfield TM, Saeed SA. An update on mindfulness meditation as a self-help treatment for anxiety and depression. Psychol Res Behav Ma. 2012;5:131-141. doi:10.2147/PRBM.S34937

25. Thompson RW, Arnkoff DB, Glass CR. Conceptualizing mindfulness and acceptance as components of psychological resilience to trauma. Trauma Violence Abuse. 2011;12(4):220-235. doi:10.1177/152483 8011416375 
26. Chen JH, Tsai PH, Lin YC, Chen CK, Chen CY. Mindfulness training enhances flow state and mental health among baseball players in Taiwan. Psychol Res Behav Manage. 2019;12:15-21. doi:10.2147/ PRBM.S188734

27. Shapiro SL, Carlson LE, Astin JA, Freedman B. Mechanisms of mindfulness. J Clin Psychol. 2006;62(3):373-386. doi:10.1002/ jclp. 20237

28. Xu W, Rodriguez MA, Zhang Q, Liu X. The mediating effect of self-acceptance in the relationship between mindfulness and peace of mind. Mindfulness. 2015;6(4):797-802. doi:10.1007/s12671-014-0319-x

29. Lee Y, Lin Y, Huang C, Fredrickson BL. The construct and measurement of peace of mind. J Happiness Stud. 2013;14(2):571-590. doi:10.1007/s10902-012-9343-5

30. Desbordes G, Gard T, Hoge EA, et al. Moving beyond mindfulness: defining equanimity as an outcome measure in meditation and contemplative research. Mindfulness. 2015;6(2):356-372. doi:10.1007/ s12671-013-0269-8

31. Liu X, Xu W, Wang Y, et al. Can inner peace be improved by mindfulness training: a randomized controlled trial. Stress Health. 2015;31(3):245-254. doi:10.1002/smi.2551

32. Ren J, Huang L, Zhang Z. Meditation makes a peaceful state of mind: people's positive and negative emotional response can be reduced by meditation training. Acta Psychol Sin. 2012;44(10):1339-1348. doi:10.3724/SP.J.1041.2012.01339

33. Wallace BA, Shapiro SL. Mental balance and well-being: building bridges between buddhism and western psychology. Am Psycho. 2006;7(61):690-701. doi:10.1037/0003-066X.61.7.690

34. Fredrickson BL, Branigan C. Positive emotions broaden the scope of attention and thought-action repertoires. Cogn Emot. 2005;19 (3):313-332. doi:10.1080/02699930441000238

35. Pepping CA, O' Donovan A, Davis PJ. The differential relationship between mindfulness and attachment in experienced and inexperienced meditators. Mindfulness. 2014;5(4):392-399. doi:10.1007/ s12671-012-0193-3

36. Wittmann M, Otten S, Schã Tz E, et al. Subjective expansion of extended time-spans in experienced meditators. Front Psychol. 2015;5:1586. doi:10.3389/fpsyg.2014.01586
37. Kemper KJ, Khirallah M. Acute effects of online mind-body skills training on resilience, mindfulness, and empathy. J Evid Based Complementary Altern Med. 2015;20(4):247-253. doi:10.1177/ 2156587215575816

38. Baer RA, Smith GT, Lykins E, et al. Construct validity of the five facet mindfulness questionnaire in meditating and nonmeditating samples. Assessment. 2008;15(3):329-342. doi:10.1177/1073191107313003

39. Deng YQ, Li S, Tang YY, Zhu LH, Ryan R, Brown K. Psychometric properties of the Chinese translation of the Mindful Attention Awareness Scale (MAAS). Mindfulness. 2012;3(1):10-14. doi:10.1007/s12671011-0074-1

40. Yu X, Zhang J. A comparison between the Chinese version of ego-resiliency scale and connor-davidson resilience scale. Psychol Sci. 2007;30:1169-1171.

41. Sircova A, van de Vijver FJ, Osin E, et al. A global look at time: a 24-country study of the equivalence of the zimbardo time perspective inventory. SAGE Open. 2014;1(4):1-12.

42. Wu Y, Wen Z.Item parceling strategies in structural equation modeling. Adv Psychol Sci. 2011;19(12):1859-1867.

43. Wang Y, Xin TT, Liu XH, Zhang Y, Lu HH, Zhai YB. Mindfulness can reduce automatic responding: evidences from Stroop task and prospective memory task. Acta Psychol Sin. 2012;44(9):1180-1188. doi:10.3724/SP.J.1041.2012.01180

44. Bishop SR. Mindfulness: a proposed operational definition. Clin Psychol Sci Pract. 2004;11(3):230-241.

45. Bercovitz K, Pagnini F, Phillips D, Langer E. Utilizing a creative task to assess langerian mindfulness. Creat Res J. 2017;29(2):194-199. doi:10.1080/10400419.2017.1304080

46. Sword RM, Sword RKM, Brunskill SR, Zimbardo PG. Time perspective therapy: a new time-based metaphor therapy for PTSD. J Loss Trauma. 2014;19(3):197-201. doi:10.1080/15325024.2013.763632

47. Zimbardo PG, Sword R, Sword R. The time cure: overcoming PTSD with the new psychology of time perspective therapy. J Fam Ther. 2012;41(5):452-454.
Psychology Research and Behavior Management

\section{Publish your work in this journal}

Psychology Research and Behavior Management is an international, peer-reviewed, open access journal focusing on the science of psychology and its application in behavior management to develop improved outcomes in the clinical, educational, sports and business arenas. Specific topics covered in the journal include: Neuroscience, memory and decision making; Behavior modification and management; Clinica applications; Business and sports performance management; Social and developmental studies; Animal studies. The manuscript management system is completely online and includes a very quick and fair peer-review system, which is all easy to use. Visit http://www. dovepress.com/testimonials.php to read real quotes from published authors. 